Théologiques

Théologiques

\title{
Mystique du néant et śūnyatā selon la perspective de l'École de Kyōto
}

\section{Giancarlo Vianello}

Volume 20, numéro 1-2, 2012

Les philosophes de l’École de Kyōto et la théologie

URI : https://id.erudit.org/iderudit/1018862ar

DOI : https://doi.org/10.7202/1018862ar

Aller au sommaire du numéro

\section{Éditeur(s)}

Faculté de théologie et de sciences des religions, Université de Montréal

\section{ISSN}

1188-7109 (imprimé)

1492-1413 (numérique)

Découvrir la revue

\section{Citer cet article}

Vianello, G. (2012). Mystique du néant et śūnyatā selon la perspective de l'École de Kyōto. Théologiques, 20(1-2), 297-312. https://doi.org/10.7202/1018862ar
Résumé de l'article

Au début des années 1960, Ueda Shizuteru alla étudier à Marburg, sous la direction d'Ernst Benz. Le travail de cette époque se concentra sur la spéculation de Maître Eckhart et sur la comparaison avec le bouddhisme. En particulier, l'étude de Ueda fit émerger deux différentes approches de la question du néant. La mystique du néant et la śunnyatā bouddhiste furent analysées dans leurs aspects constitutifs. Cet approfondissement se révèle précieux, non seulement comme étude interculturelle, mais surtout comme contribution à l'interprétation du contexte nihiliste que l'Europe et le Japon se retrouvent à partager et auquel ces derniers ont affaire. Les observations de Ueda et, en général, des auteurs de l’École de Kyōto, offrent une perspective totalement autre dans la façon de traiter les noeuds ontologiques et théologiques de la pensée occidentale. 


\title{
Mystique du néant et śūnyatā selon la perspective de l’École de Kyōto
}

\author{
Giancarlo Vianello*
}

Au début des années 1960, Ueda Shizuteru, un philosophe déjà affirmé de l'École de Kyōto, se rendit en Allemagne pour étudier à Marburg, sous la direction de Ernst Benz. Il ressentait en effet le besoin d'une recherche ad fontes, focalisée sur un approfondissement philologique et linguistique, lui permettant une interprétation, d'un point de vue japonais, de l'itinéraire spéculatif de Maître Eckhart. Il s'agissait en outre d'une recherche supportée par un grand sens de l'humilité et une connaissance solide de l'allemand, du Mittelhochdeutsch et du latin médiéval.

Maître Eckhart est une figure qui a toujours exercé une attraction particulière sur les penseurs japonais, tant à cause d'une certaine assonance entre sa spéculation et la pratique du bouddhisme zen, que parce qu'il représente une articulation centrale de la pensée occidentale. Son influence, en effet, se projette à travers les siècles, du milieu proprement rhénan, jusqu'à inspirer le quiétisme d'un Angélus Silesius, pour aboutir au Schelling des conférences d'Erlachen ${ }^{1}$. Mais ce sont surtout la richesse et l'hétérogénéité des sources d'Eckhart qui attirèrent le chercheur japonais. Le mystique dominicain fut un collecteur des influx qui dérivent du christianisme, mais aussi d'autres traditions. Ce matériel fut structuré de façon organique dans une vision cohérente. Parmi les éléments les plus significatifs qui l'influencèrent, on retrouve des conceptions dérivant de la gnose de Basile, du néoplatonisme, de la spéculation mystique hébraïque et cabalis-

\footnotetext{
* Giancarlo Vianello est membre fondateur du Centro Studi Maitreya (Venise) et chercheur de l'École de Kyōto. Il a récemment publié (2011), Colligite fragmenta. La questione del nulla, Rubbettino.

1. Une liste schématique des mystiques et des théologiens influencés directement par Maître Eckhart peut comprendre, outre Henri Suso (1295-1366) et Jean Tauler (1300-1361), Jan van Ruusbroec (1293-1381), Nicolas de Cuse (1401-1464), Valentin Weigel (1533-1588), Jakob Böhme (1575-1624), Daniel Czepko (16051660) et enfin Angelus Silesius (1624-1677).
} 
tique, du pseudo Denys et de Scot Eurigène (Nambara 1960). De plus, le néoplatonisme était pratiqué au sein du Studium generale des dominicains de Cologne, tant dans sa dérivation augustinienne, à travers Plotin, qu'à travers le pseudo Denys, par le biais de Proclus.

Les thèmes de la Gottheit et de la théologie négative, qui représentent peut-être le point central de la spéculation eckartienne, dérivent surtout de ce dernier filon (Koch 1948). Ueda fut fasciné par ce réseau conceptuel qui innerve la pensée occidentale et il orienta sa recherche vers une interprétation de la mystique eckartienne selon sa perspective de bouddhiste japonais. Sa recherche eut une influence sur la critique eckartienne, car, d'un point de vue bouddhiste, donc radicalement autre, elle se révéla capable de saisir ce qui échappait à des auteurs qui se déplaçaient à l'intérieur d'une histoire spirituelle composée aussi de contrapositions et idiosyncrasies. Avec lui, s'ouvrit un filon critique auquel se rallieront des chercheurs comme Schürmann (1978), Haas $(1979 ; 1994)$ et Largier (1992).

Le travail de Ueda se concrétisa dans une thèse de doctorat: La naissance de Dieu dans l'âme et l'irruption dans le Divin: l'anthropologie mystique de Maître Eckhart confrontée à la mystique du Bouddhisme Zen (1965), parue avec une stimulante préface de Ernst Benz. Une série d'essais sur ce thème suivirent cette publication $(1965 ; 1967 ; 1977 ; 1989)$.

\section{Une lecture bouddhiste de Maître Eckhart}

Ueda saisit dans Eckhart une tension vers l'absolu qu'il essaie d'exprimer avec n'importe quel instrument conceptuel, d'abord ceux de la dérivation néoplatonicienne. En même temps, il est fortement enraciné dans les formes de sa propre foi. En lui, doctrine trinitaire et apophatisme néoplatonicien constituent les deux moments d'une construction conceptuelle cohérente.

Le point de départ de sa théologie mystique est le thème du détachement, la Gelassenheit. Se détacher de tout ce qui est inessentiel, et pour cela aliénant, produit la liberté. S'abandonner soi-même et abandonner toutes les choses conduit au fond de l'âme, dans ce lieu purifié dans lequel les contraires, les visions opposées et partielles n'opèrent plus. Le fond de l'âme est Dieu même, l'Abgrund, une profondeur insondable qui constitue l'origine. Dans ce lieu, une fois surpassés chaque nombre et chaque multiplicité, âme et Dieu se compénètrent: l'homme est anéanti comme sujet et devient esprit dans l'esprit. L'abysse insondable du divin se place au-delà de toute détermination: pour cela il est sans pourquoi: il échappe à n'im- 
porte quelles logique et relation, d'abord celles d'ordre linguistique. En s'annulant et en se compénétrant avec Dieu, l'âme aussi devient sans pourquoi, elle coupe ses liens avec le monde et ses catégories logiques.

Ueda définit la théologie de Maître Eckhart comme «une mystique de l'infinité avec un fondement théiste» (Ueda 1965, 143, nous traduisons). Dans l'œuvre du Maître, on peut reconnaître un désaccord entre la tension extrême - aussi hétérodoxe - vers l'infinitude et l'attachement aux formes de sa propre foi. Ce désaccord s'articule autour des deux thèmes qui caractérisent la spéculation eckartienne: la naissance de Dieu dans l'âme et l'irruption dans le divin.

Le thème de la naissance de Dieu dans l'âme, qui exprime le fondement trinitaire de la construction mystique, est la base sur laquelle se développe la spéculation suivante. Eckhart, comme on l'a dit, part de la doctrine trinitaire. Le Père génère, comme c'est implicite dans sa propre nature. Il génère donc le Fils dans sa personne même, mais aussi dans chaque âme, dans l'éternité. Pour le dire avec les mots d'Eckhart: «Pater generat suum Filium in anima eodem modo sicut Ipse generat Eum in aeterno non ali$t_{e r}^{2} »$ (Proc. Col. II, 95, LW V, 340, 25-26, Quint 1936; Daniels 1923, 53).

La nature humaine est connectée à la nature divine, parce que Dieu s'incarne dans tous les hommes. L'âme est l'image de Dieu et elle est coparticipante de la nature divine. Elle doit donc se séparer de tout pour rejoindre Dieu: quand chaque individu s'oublie soi-même et, dans le silence, devient la parole même, il vit l'expérience de l'ablatio alteritatis, de la fin de la séparation et de l'aliénation.

Sans finalité et sans pourquoi, la vie acquiert un sens plus profond, à l'instant où on s'évade de la prison du moi, de l'existence individuelle. L'âme, en tant qu'image de Dieu, doit réaliser sa nature en se vidant du moi et en rejoignant Dieu. À ce point, la dynamique mystique s'ouvre à de nouveaux développements.

L'abandon à Dieu, toutefois, signifie s'abandonner à une représentation humaine. Dieu est un terme qui est utilisé par nécessité. Eckhart comprend une essence de Dieu qui transcende Dieu même: au-delà des trois personnes de la Trinité se pose une Gottheit transcendante de Dieu et de ses articulations.

Ce noyau originaire se connote tout de suite comme impersonnel et indéfini et il en vient à être en quelque sorte exprimé avec les instruments

2. «Le Père engendre son Fils dans l'âme de la manière même dont Il l'engendre dans l'éternité, et pas autrement.» 
de la théologie négative. Il est pur néant, au sens où il est au-dessous de tous les êtres. L'âme, en s'enfonçant dans la Gottheit, revient à sa propre nature: elle abandonne Dieu pour s'unir à l'essence de Dieu.

Comme le dit Maître Eckhart: «Summum quod homo relinquere posset est quod ipse Deum propter Deum relinquat ${ }^{3}$ "(Daniels 1923, 33). L'irruption dans la Gottheit complète la dynamique mystique d'Eckhart. L'âme, qui est générée avec la nature divine, se réalise en abandonnant le monde et en s'unissant à Dieu, pour ensuite atteindre l'essence de Dieu. À ce point, ayant expérimenté le néant, elle est capable de revenir à la réalité quotidienne.

Maître Eckhart porte à ses conséquences extrêmes l'ontologie plotinienne du néant. Ce dernier devient le visage de Dieu. Dans le célèbre Sermon LXXI (Pr. 71, DW III, p. 211, 5-7; Quint 1936), il affirme: «Lorsqu'il [saint Paul] se releva de terre, les yeux ouverts, il vit le néant et le néant était Dieu» (nous traduisons). Le passage des Actes des Apôtres $(9,8)$, «Surrexit autem Saulus de terra apertisque oculis nihil videbat ${ }^{4}$ », est interprété avec une audace inouie. Dieu est le néant duquel l'être jaillit, il est les ténèbres desquelles jaillit le voir. Saint Paul, ne voyant plus rien, a la vision de l'origine. Avec cette vision, s'annihilent toutes les autres: le monde des créatures est réduit au néant qu'il est et donc il disparaît. Le néant de l'origine est, en même temps, l'origine de Dieu, de ce que les humains conçoivent comme Dieu. Ce dernier est, plus proprement, le néant des choses qui sont. Il existe précédemment à l'être et il en est dénué, comme il est dénué de n'importe quel autre prédicat. L'absolu doit être au-delà de la relation Créateur/créé, au-delà de chaque relation et au-delà de chaque "pourquoi ": âbne warumbe, sans pourquoi. L'absolu est sans pourquoi, il exprime naturaliter et sans délibération sa propre divinité. La Gottheit est néant, absolue négation de toutes distinctions. Elle est transcendance extrême. En tant que tel, le néant divin souligne son «incomparabilité » absolue par rapport à chaque être.

Symétrique au "grand Néant», se situe le «Néant infime », la créature. La créature en soi est un néant, le seul être auquel elle a droit étant celui reçu de Dieu. La créature vit dans un état de dépendance, de précarité, de contingence. Sans Dieu, sans l'être octroyé par lui, elle s'anéantirait à l'instant. Elle existe seulement ex nibilo et ex Deo. Elle est traversée par une

3. «L'abandon le plus élevé et le plus total que l'homme puisse faire est d'abandonner Dieu pour Dieu.»

4. «Paul se leva de terre et les yeux ouverts il ne voyait rien.» 
nullité radicale. Cette nullité, qui constitue l'essence du monde, doit être abandonnée: il faut aboutir à un détachement (Abgeschiedenheit) de la créature pour arriver à Dieu. Paradoxalement, il faut s'abandonner soimême et devenir pur néant. Autrement dit, il faut devenir le néant du néant: une fois abandonnée la créature, avec l'âme réduite à néant, on procède vers le Néant «superessentiel».

En tant que néant, en tant que libre de toutes choses, dont du désir de Dieu, l'âme est transcendée par une plus haute négation. Dans la Gottheit, qui est au-delà de Dieu, l'humain procède au-delà de l'humain et devient une créature incréée. Cette union dans le néant dépasse le dualisme entre Dieu et la créature. L'ego, une fois traversé le seuil du néant, s'unifie avec sa propre origine. En s'annulant dans l'abysse du néant, on se place dans le lieu qui précède toutes déterminations, y compris Dieu. Les êtres humains veulent l'être et veulent Dieu, ils poursuivent en vain ces figures ontologiques. En abandonnant la volonté et en se laissant aller au néant, on rejoint au contraire ce qu'on a poursuivi en vain.

\section{2. Śūnyatā et perspective non duale}

Ueda considère la dynamique mystique d'Eckhart similaire, sous plusieurs aspects, à celle du bouddhisme zen, surtout en ce qui concerne l'ultratranscendance et le double mouvement qui va de la réalité au néant et, de nouveau, du néant à la réalité. Ce qui sépare les deux expériences est le fait que le bouddhisme, dans toutes ses écoles et articulations, ne reconnaît pas le théisme personnel comme son fondement nécessaire. Au contraire, c'est notamment le zen qui s'en distancia en refusant toute construction mentale qui tend à créer indûment des figures métaphysiques. "Si tu rencontres Bouddha, tue-le! », récite un provocateur et célèbre principe zen, indiquant que le Bouddha et sa doctrine sont des instruments pour la libération et ne peuvent pas être transformés en fétiches, en présences qui dévient de l'acquisition de la vacuité. Dans le domaine zen, on utilise souvent la métaphore du radeau, qui est un instrument apte à gagner l'autre bord et que l'on doit abandonner une fois la rive atteinte. Selon la lecture d'Eckhart faite par Ueda, celui-ci, dans la phase du retour au quotidien, transite à nouveau par la doctrine trinitaire, qui est son fondement, et par ses représentations, démontrant avec cela, une radicalité mineure par rapport au zen.

Les observations de Ueda concernant la spéculation mystique d'Eckhart et la pratique du zen renvoient à deux réalités dont la signification 
est très éloignée: la śūnyatā bouddhiste et la mystique du néant, qui naissent et se développent dans des milieux culturels et spirituels sans relations entre eux et qui, toutefois, sont d'une certaine manière en relation avec la question du néant. Évidemment, il s'agit de deux approches très différentes de ce thème.

La pensée orientale, à la différence de celle qui s'est développée en Occident, a en général une prédisposition naturelle pour une option méontologique qui privilégie la vacuité plutôt que l'être. C'est la raison pour laquelle on peut parler de vacuité orientale. Toutefois, le courant qui a défini et articulé cette perspective avec une plus grande netteté est, à mon avis, le bouddhisme, surtout dans sa version du Mādhyamika. Ce sont surtout les sūtra de la littérature de la Prajñāpāramitā qui affirmeront la vacuité.

Le thème central de ces textes et de leurs commentaires est qu'il n'existe ni changement, ni origine, ni cessation. Le réel n'est ni un ni plusieurs; ni ātman (doté de soi) ni anātman (privé de soi). Il s'agit là de spéculations produites par l'ignorance. Le réel est absolument dépourvu de ces constructions conceptuelles. Il transcende la pensée et il ne peut être saisi que par la connaissance non duelle, la prajñā. La vacuité (śūnyatā) n'est pas une autre conception, mais l'absence de toute conception. En fait, la littérature de la Prajūāpāramitā se centra sur le thème de la śūnyatā, qui est la négation de toutes les notions empiriques et de toutes les théories spéculatives.

L'affirmation centrale de la littérature de la Prajūāpāramitā: «la forme est le vide, le vide est la forme ", ne doit pas être entendue comme identité, mais comme une inclusion réciproque, la coexistence des phénomènes et de la vacuité. Cette dernière ne doit donc pas être pensée comme un principe abstrait ou en quelque sorte fondateur, mais comme co-appartenant aux phénomènes.

Non seulement la śūnyatā ne peut pas être entendue comme le fondement du réel, mais encore, elle ne peut être hypostasiée; elle ne doit pas se transformer en principe métaphysique apte à ouvrir sur une perspective dualiste. Dans les textes de la Prajūāpāramitā, l'action de la śūnyatā ne se limite pas à annihiler le soi et les phénomènes, mais à annihiler la vacuité même. Une des dix-huit formes de vacuité (Conze 1973, 165) est la śūnyatāśūnyatā, c'est-à-dire la vacuité de la vacuité. La vacuité s'annihile ellemême pour permettre à la forme d'apparaître, dans un processus continu de naissance et de mort. 
Ce qui est affirmé dans la littérature de la Prajñāpāramitā est par la suite organisé logiquement et philosophiquement par Nāgārjuna, le fondateur de l'école Mādhyamika. Dans son Madhyamakakārikā (Traité du milieu), il vise à démolir logiquement les catégories illusoires, que par convention on appelle réalité, et à rechercher cet absolu inaccessible indiqué par la Prajñāāaramitā. Avec une rigueur implacable, il démontre la vacuité de l'esprit, de la substance et des attributs, des causes et des effets. Il n'y a rien de réel. Il n'y a ni vérité ni fausseté. Il n'y a que des phénomènes transitoires, qui se relient entre eux grâce à la relation. Nāgārjuna refuse toutes les visions, en opérant à travers une reductio ad absurdum. Produire des visions, en effet, a une fonction de couverture et obtient le même résultat que cacher et déformer. Nous vivons possédés par nos visions. Le Mādhyamika par contre essaie de les compromettre à travers la śunnyatā, qui met en lumière leur nature vide. Les catégories sont des artifices conceptuels qui ont tendance à relativiser le réel et elles deviennent donc une falsification de ce dernier. En déconceptualisant l'esprit et en se démettant des visions et de leurs incrustations, on se rend apte à comprendre la réalité à travers la prajīā, qui est connaissance non duelle sans contenu. Elle est intuition immédiate de la totalité. Elle est en outre une connaissance dont on n'a pas conscience, car si c'était le cas, on poserait de nouveau une dualité entre connaissant et connu.

Selon le Mādhyamika, la réalité suprême est ineffable et, étant au-delà du langage, on ne peut la définir ni comme vide, ni comme non vide, ni les deux choses à la fois, ni aucune des deux. Elle est, par convention, définie par le terme śúnyatā, qui ne doit pas être entendu dans une optique négative, car absolu et relatif se compénètrent.

\section{Mystique du néant et marginalisation du néant}

Du côté occidental, on trouve en revanche une approche mystique qui traite de la question du néant dans une perspective évidemment très différente. La mystique du néant, dont Eckhart est l'un des représentants les plus significatifs, se développa en Occident à partir de l'enseignement de Plotin.

C'est Plotin qui, en particulier, identifia la matière et le néant (Ennéades, I, 8,5; II, 4; III, 6,6-7). L'être, en tant que multiplicité, est pénétré par le néant et le principe absolu, l'Un, transcende l'être même. L’Un est $\mu$ 门’óvv, surabondance d'être (Ennéades, VI, 9,5). En ce sens, il est opposé à la matière, qui est caractérisée par une carence d'être. La matière 
est l'élément vague et indéfinissable, mais éternel, avec lequel le kosmos est modelé. À l'extrême opposé se trouve l'Un, duquel on ne peut rien affirmer et qui ne nécessite rien. En cela, il est apparenté au néant, car le néant est le seul attribut qui lui peut convenir. De plus, la puissance génératrice doit forcément rester au-delà du généré: l'Un modèle l'identité de la réalité, restant toutefois infiniment autre. Il est le néant des choses qui existent.

Le néant, plus que l'être, semble être l'abyssale dimension de l'Un. Seulement, il est possible d'avoir l'intuition de la présence de l'Un en faisant abstraction de ce qu'il y a d'inessentiel dans les étants. Le principe générateur est beauté et liberté sans pourquoi, qui conforme le domaine de l'être. Pour cela, il devient possible, en abandonnant l'inessentiel de l'être, d'avoir l'intuition dans le monde également de la beauté et de la liberté, qui sont des formes qui descendent du néant. L'expérience extatique qui conduit à l'intuition de l'Un est in primis un processus de purification. L'Un laisse être la forme, qui toutefois est capturée par la matière qui l'emprisonne, la disperse et la rend amorphe. De cela, naît la double méontologie de Plotin. Il s'agit, d'un côté, du néant, c'est-à-dire de la puissance de l'Un et, de l'autre, de la puissance opposée à la matière. Ce double néant représente le domaine dans lequel se joue le drame humain.

La mystique du néant se développa dans un milieu culturel et philosophique caractérisé par la polarisation d'une liaison être/néant et par une forte propension pour le pôle de l'être.

Ce même filon mystique, auquel Eckhart appartient, se développa à travers les suspicions, les censures et les autocensures, et fut toujours vu de manière suspecte par les autorités ecclésiastiques, fussent-elles catholiques ou luthériennes. En Occident, le néant, quand il est présent, fait scandale et il est marginalisé. En tout cas, il doit faire face à une vision métaphysique de type dualiste et avec une orthodoxie déséquilibrée sur le versant de l'être.

Le fait est que la pensée occidentale est marquée aux origines par les affirmations radicales de Parménide, qui affirme explicitement que le néant n'est pas et est absolument autre que l'être (Physis, 117, 2). Parménide semble vouloir prendre ses distances avec le monde tragique. La tragédie grecque exprime, en premier lieu, l'ambiguïté de l'existence humaine, enserrée entre le désir de la vie et la nécessité de la mort, de l'annihilation comme destin. Parménide fuit cette ambivalence. Il veut placer la vérité des choses à l'intérieur d'un horizon éternel et immuable. S'il y a autre chose en plus de l'être, cela est autre que l'être et n'existe donc pas. En niant le néant, Parménide nie la contradiction et l'énigme. Il substitue à la méon- 
tologie tragique, fondée sur l'ambiguïté de l'événement, une métaphysique de l'être qui bannit tout dualisme. L'être, en tant que non généré, ne peut périr; il est entier, unique, immobile et sans fin. Ce qui a l'existence ne peut pas la perdre.

La perspective parménidienne signa l'itinéraire de la pensée occidentale dans le sens de la prééminence de l'être. Des auteurs comme Maître Eckhart, qui élaborent une mystique du néant, se trouvent donc à opérer à l'intérieur d'un contexte spéculatif opposé.

En général, la philosophie européenne exorcise le néant, qui est pensé comme l'opposé de l'être, donc dans la perspective de l'être et en termes négatifs. Autrement dit, ce dernier est conçu comme un manque de quelque chose qui naturellement devrait y être. Un tel manque peut être limité à un être ou à la totalité des êtres. Dans cette double nature, émerge la différence entre un néant relatif et un néant absolu.

Comme on le disait, la philosophie marginalise le néant, avec des pointes de refus particulièrement fortes chez des auteurs comme Leibniz, Bergson, Russell, et chez les néopositivistes. Cette attitude a toutefois changé récemment, avec des auteurs qui, comme les existentialistes et Heidegger, prirent conscience de l'achèvement de l'itinéraire métaphysique occidental et de la crise produite par la dérive nihiliste.

\section{La crise nihiliste et le retour de la question du néant}

Selon Heidegger, la métaphysique occidentale s'est développée sur un plan ontique, au niveau du étant, et elle est donc essentiellement nihiliste. Elle a accompli un itinéraire qui devait nécessairement aboutir au nihilisme (Heidegger 1950). Nihiliste est, en effet, l'horizon dans lequel elle se trouve à penser. De plus, ce n'est pas un hasard si, face à la crise mémorable de la métaphysique en Europe, émerge une sensibilité différente à l'égard de la question du néant. Le nihilisme est un phénomène historique qui implique la civilisation européenne et qui a vu s'éroder, avec une rapidité pathologique, ses valeurs fondatrices. Il s'agit en outre d'un phénomène qui a marqué l'histoire spirituelle et matérielle du $\mathrm{xx}^{\mathrm{e}}$ siècle. Depuis qu'on a ressenti sa présence corrosive, on a commencé à avoir contextuellement conscience de la conclusion d'un cycle de civilisation, de notre manière de vivre dans une époque de décadence. Par la suite, rapidement, ont suivi la crise, c'est-à-dire la réflexion critique sur la nature de la décadence, le débat sur la sortie possible du nihilisme, la résurgence du thème du néant 
dans la pensée occidentale, la maîtrise hors de tout contrôle de la technique, la mondialisation du nihilisme à l'échelle planétaire.

Le visage avec lequel le nihilisme est saisi par Nietzsche est celui de la dévaluation des valeurs, de l'Entwertung. Les valeurs ne se corrodent pas à long terme, mais elles se désagrègent dans l'immédiat; elles perdent leur consistance avec une facilité pathologique. Non seulement elles sont inaptes à une quelconque fonction, mais elles ouvrent la route à une instabilité et à une incohérence répandues. Quand il n'existe plus de valeurs générales aptes à centraliser et à tenir unies les instances individuelles de la société, s'affirme la parcellisation des valeurs particulières, individuelles. La volonté de puissance et l'accroissement de ses propres potentialités émergent alors dans notre époque comme seul fondement possible des valeurs. En même temps, de la détérioration des valeurs émerge la conscience de la relativité de celles-ci et s'ouvre une réflexion à propos de l'autre côté de l'être: le néant. Le nihilisme consiste donc aussi, étymologiquement, à sonder le fondement du néant.

\section{Le nihilisme au Japon}

Le retour de la question du néant à travers la crise nihiliste n'impliqua pas seulement la pensée occidentale. Avec l'expansion de la modernité et de la technique à un niveau planétaire, le nihilisme même, qui naquit comme phénomène proprement européen, s'enracina sous d'autres cieux et il atterrit au Japon, dans le contexte de la modernité. La technique, que le Japon s'est approprieé, est toutefois un fragment de la culture occidentale, qui s'est délocalisée en perdant ses racines. Le Pays du Soleil Levant est sans doute le lieu qui a été le plus influencé par la technique. Contextuellement, c'est un pays doté d'une identité culturelle forte et bien structurée, construite à travers le syndrome de l'insulaire. Cette identité a accepté des stimulations culturelles provenant de la Chine: l'écriture, l'éthique confucéenne, le bouddhisme. Elle a pourtant remodelé ces influences fondamentales à l'intérieur d'un complexe culturel entièrement japonais.

Dans le cas de l'acceptation hypertrophiée de la technique occidentale, les choses sont allées d'une façon différente, puisque la modernité et la technique ont aussi amené avec elles leurs fruits empoisonnés, comme le nihilisme. Les deux sont entrées de façon envahissante dans la société japonaise et ont démoli des équilibres et des rapports de force; elles ont dévasté les modes de vie, les expressions culturelles, les modalités du vivre social. Elles ont surtout provoqué la crise des fondements culturels et des 
énergies spirituelles japonaises, imposant un processus très profond d'aliénation. Comme on l'a dit, la technique représente un fragment qui a pris son autonomie par rapport à la modernité, mais qui, toutefois, est né et a grandi dans ce processus historique. Nous, Occidentaux, nous avons clairement conscience des étapes de ce processus: civilisation communale et mercantile, Renaissance, découvertes géographiques, esprit scientifique, Réforme, empirisme et rationalisme, Lumières, dialectique, révolution industrielle. Il s'agit de notre histoire. Ce n'est pourtant pas l'histoire du Japon. Comme le disait Karl Löwith (Löwith 1983, 588), la modernité pour le Japon est une réalité indiscutée et une impossibilité. D’un point de vue logique et formel, il s'agit d'un oxymore. D'un point de vue pratique, c'est l'expansion de la technique, au nom du principe de prestation, et la marginalisation de la culture traditionnelle. On peut facilement imaginer quels effets cela a pu avoir dans une culture caractérisée par la tendance à métaboliser complètement les prêts culturels provenant de l'extérieur. On peut aussi facilement imaginer quelles énergies intellectuelles une telle situation a miess en mouvement, dans la tentative de récupérer un sens pour la modernité japonaise. Le problème herméneutique de la relation entre Japon et Occident reste, et ce n'est pas un hasard, encore ouvert.

Sur ce problème se concentra la réflexion de l'École de Kyōto, c'est-àdire de ce groupe de philosophes qui continua l'œuvre spéculative de Nishida Kitarō. Il s'agit d'un groupe de penseurs qui, malgré des différences et une certaine dialectique interne, sont réunis par l'adhésion à une perspective ontologique qui pose le néant absolu comme élément constitutif de la réalité. L'École de Kyōto a opéré une adéquation de la pensée traditionnelle japonaise d'ascendance bouddhiste avec les instruments de la philosophie, rendant possible une confrontation et un dialogue. Quoique les différents penseurs appartiennent à différenets écoles du bouddhisme, ils ont en commun l'arrière-fond spéculatif du Mãdhyamika et comme point de référence la śūnyatā, même si elle est déclinée suivant différentes acceptions.

Il est significatif que les réflexions entreprises par l'École de Kyōto soient advenues à une époque où la pensée de l'Orient et celle de l'Occident cessèrent de se développer parallèlement. Des phénomènes intrinsèquement différents les uns des autres, comme la philosophie et le bouddhisme, en arrivèrent à faire partie du même système culturel planétaire. L'occidentalisation à une échelle planétaire, qui est désormais consommée, a créé un cadre dans lequel les différentes formes culturelles ont dû se repositionner, insérées qu'elles sont dans un complexe homogène, qui en 
restructure les particularités. Cet amalgame, qui nie l'altérité, a pris l'aspect de la modernité, d'une modernité dans sa phase terminale, qui a épuisé les narrations légitimantes. D'un point de vue culturel, on en est à une contamination diffusée et uniformisante qui rend impossible le dialogue, lequel ne pourrait s'effectuer qu'à partir d'une altérité consciente.

L'École de Kyōto a eu le mérite d'avoir repensé de manière organique la radicalité originaire japonaise et d'en avoir redéfini l'identité. Elle a représenté un repère important pour la culture japonaise, en phase de transition. Contextuellement, la leçon de l'École de Kyōto revêt une importance décisive aussi pour la pensée occidentale, car elle arrive à traiter des thèmes communs avec une voix autre. Les philosophes de cette école maîtrisent un double langage et embrassent un double horizon. Ils sont insérés dans la modernité et ils tirent leurs racines de la pensée traditionnelle bouddhiste et de son ontologie. Ils sont surtout capables d'exprimer des observations originelles sur le thème de l'être et du néant. De telles contributions sont en train de se révéler très précieuses pour la philosophie occidentale en pleine dérive nihiliste. À partir des difficultés théoriques de la pensée occidentale, arrivée à son stade nihiliste, l'École de Kyōto arrive à parler, avec le même langage, d'un autre horizon ontologique. Elle arrive en outre à intervenir sur les instances spéculatives particulières, sous un angle différent et en utilisant un langage philosophique formellement irréprochable.

Toutefois, la contribution majeure que l'École de Kyōto peut offrir à la pensée globale est, à mon avis, sa capacité d'adopter une optique non duelle, en refusant les polarisations, qui ont en revanche caractérisé la philosophie européenne.

Être et néant, dans le sillage de la tradition du Mādhyamika, sont vécus comme parties d'une unité harmonieuse plutôt que polarisés dans une optique positif/négatif. De plus, l'inspiration bouddhiste de l'école porte à une attitude de connaissance de la fragilité du réel, justement impermanent et vide, et au recouvrement du sens du mystère et de la stupeur face au monde. Par contre, il y a eu en Occident un processus d'objectivation de l'étant qui a amené à considérer la nature comme une matière modelable. À un moment où la philosophie prend de plus en plus des accents poétiques ou mystiques, j'estime être d'utilité extrême de constituer un trésor avec cette autre sensibilité, qui offre une lecture plus harmonieuse de la réalité. 


\section{Mystique du néant et śūnyatā}

Cette sensibilité, pour donner un exemple concret, peut être relevée dans le texte de Ueda avec lequel a démarré cet article, notamment lorsqu'il commente, le célèbre vers d'Angélus Silesius: «La rose est sans pourquoi, elle fleurit parce qu'elle fleurit» (Silesius 1984, 289, nous traduisons). Dans ce vers, la rose, qui est une créature, est mise en relation en tant que sans pourquoi avec Dieu: son fleurir est une manifestation de Dieu. La rose, comme toute créature, est animée par l'être de Dieu et est en conséquence sans pourquoi. Ueda observe qu'Eckhart considérait Dieu comme l'être de la nature, comme ce qui en permet l'existence à travers la creatio ex nibilo. La rose, en tant que créature, est pur néant. Elle existe seulement grâce à l'action divine. En abandonnant son être néant, elle exprime un événement divin. Elle est une incarnation de Dieu. Dans cette perspective, toutefois, Dieu apparaît en relation avec une créature. Cela, en plus de promouvoir une mystique de la nature, comporte une dualité. C'est la raison pour laquelle Dieu, qui apparaît dans la forme du sans pourquoi, n'est pas encore Dieu tel qu'il est en soi. De plus, le sans pourquoi représente un jugement humain, un prédicat, sur Dieu et sur la rose.

Il s'agit d'une pensée qui veut exprimer avec des mots humains le voilement de l'impensable être de Dieu, d'une double dualité. Le zen aurait dit seulement la rose. Cette coupure du sans pourquoi aurait signifié la totale disparition de Dieu dans le néant, le silence total de l'homme sur l'absolu et le dépassement de toute dualité. Avec cette coupure, c'est-à-dire avec la disparition de Dieu dans le néant, la rose aussi s'annihile et l'homme, en tant que créature, disparaît et renaît en Dieu, comme fils de Dieu. C'est ce que la tradition zen appelle «la Grande Mort». La rose alors devient incarnation du néant. Dieu s'est transformé en néant et le néant est devenu la rose. Le zen ne dirait jamais: "Dieu est néant». Plutôt, à la question «Qu'est-ce que Dieu? », il répondrait: «Néant». Ce faisant, il ne nierait pas Dieu, mais la question. Il nierait la dualité et la perspective anthropocentrique.

Selon Ueda, le bouddhisme zen a comporté une radicalité majeure par rapport à la perspective eckartienne. Le néant bouddhiste, la śūnyatā, n'est pas un signe qui indique un autre aspect de l'Un, mais quelque chose qui se pose au-delà de l'Un, comme le zéro transcende tout caractère numérique. Il est au-delà de toute catégorie conceptuelle. Eckhart indique Dieu en contraposition à l'humain. C'est la négation de la créature qui, en soi, est une négation, negatio negationis. Selon le maître dominicain, le terme 
«Dieu» reste central, malgré la recherche apophatique de l'essence de Dieu. L'âme écoute la parole de Dieu et répond à l'appel, mais ce faisant, elle dialogue avec Dieu. Eckhart reporte la transcendance et l'absolu sur un plan linguistique. Le bouddhisme zen, en revanche, refuse toute référence conceptuelle et linguistique et renvoie à l'ensemble non dual de la réalité. Un moine demanda à Jōshu: "Que signifie l'arrivée du patriarche de l'Occident? ». Jōshu répondit: «Un chêne devant le jardin.» L'arrivée du patriarche Bodhidharma en Chine est l'événement fondateur du zen et ce moine cherche une réponse qui donne sens à cet événement et à la vérité du zen. Symétriquement, résonne la question fondamentale de la sotériologie chrétienne: pourquoi Dieu s'est-il fait homme? (Cur Deus homo?). À la question cur Deus homo?, Eckhart répond ceci, qui sonne sous forme de question et réponse: "...und dar umbe ist er mensh geworden, daz er dich gebere sinen eingebornen sun, und nicht minner» (pourquoi Dieu s'est-il fait homme? Pour pouvoir te générer comme son fils unique, Eckhart 1955, 357, 22). Pour autant que la réponse d'Eckhart soit foudroyante, elle se déplace dans une dimension logique linguistique. Jōshu, en revanche, avec sa réponse incongrue, refuse la question et le fait que le moine se pose sur un plan linguistique. Il indique toutefois la réalité dans son entièreté.

La pratique du zen, explique Ueda, nie en même temps la question, ce qui est demandé et celui qui pose la question. Le néant est, dans ce cas, le franchissement de la dualité et il est différent de l'expression «Dieu est néant». Il s'agit d'une unité complète: «une grande boule de feu, qui brûle les différences », comme l'affirme le zen.

Chaque être est traversé par le néant, mais il est dans un rapport de relation universelle. Il ne s'annihile pas au sens strict, mais participe à la transformation continue du tout. Dans la perspective du zen, il n'est pas fermé en soi-même et il ne peut pas être défini selon les critères serrés du principe d'identité $A$ est $A$. De préférence, il est exprimé sous la modalité A est comme (soku) A, qui en souligne la nature relationnelle. Dans cette perspective, rappelle Ueda, le zen récupère une possibilité infinie de s'exprimer poétiquement et en même temps rigoureusement, sans devoir recourir à de trompeuses conceptualisations.

\section{Références}

Conze, E. (1973), The Short Prajñāpāramitā Texts, Londre, Luzac. 
DANiEls, A. (1923), "Eine lateinische Rechtfertigungsschrift des Meister Eckharts", dans Beiträge zur Geschichte der Philosophie des Mittelalters, XXXII, 5.

Eckhart, M. (1955), Deutsche Predigte und Traktate, Hg. J. Quint, Munich.

HaAs, A. M. (1979), Sermo mysticus: Studien zur Theologie und Sprache der deutschen Mystik, Fribourg, Presse de l'Université.

(1994), "Correspondances entre la pensée eckartienne et les religions orientales », dans Emilie Zum Brunn dir., Voici Maître Eckhart, Grenoble, Million, p. 374-383.

Heidegger, M. (1950), "Nietzsches Wort "Gott ist tot” ", Holzwege, Frankfurt a.M., Vittorio Klostermann.

Koch, J. (1948), Platonismus im Mittelalters. Akademische Festrede 26.5.1948 zur Universität Grundungsfeier, Krefeld, Im Scharpe, p. 7-45.

LARgier, N. (1992), "Meister Eckhart und die Osten : zur Forschunggeschichte", dans H. Stirnimann et R. Imbасн, dir., Eckhardus Theutonicus homo doctus et sanctus, Dokimion 11, Freiburger Zeitschrift für Philosophie et Theologie, Fribourg, Presse de l'Université, p. 185-204.

Löwiтh, K. (1983), «Unzulängliche Bemerkungen zum Unterschied von Orient und Okzident », dans Sämtliche Schriften, vol. 2, Weltgeschichte und Heilgeschehen. Zur Kritik der Geschichtsphilosophie, Stuttgart, J.B. Metzler.

Nambara, M. (1960), "Die Idee des absoluten Nichts in der deutschen Mystik und seine Entsprechungen im Buddhismus ", Archiv für Begriffsgeschichte. Bausteine für eine historische Wörterbuch der Philosophie, 6, p. 143-277.

Quint, J. (1936), Die deutschen und lateinischen Werke, vol. I-VI, Stuttgart/ Berlin.

Schürmann, R. (1978), "The Loss of Origin in Soto Zen and Meister Eckhart», The Tomist, 42, p. 281-312.

Silesius, A. (1984), Cherubinischer Wandersmann, Stuttgart, Reclam.

UEDA, S. (1965), Die Gottesgeburt in der Seele und der Durchbruck zur Gottheit. Die Mystische Anthropologie Meister Eckharts und ibre Konfrontation mit der Mystik des Zen Buddhismus, Gütersloh, Gütersloher Verlaghaus Gerd Mohn.

(1965), «Der Zen Buddhismus als Nicht-Mystik. Unter besonderer Berücksichtigung des Vergleichs zur Mystik Meister Eckharts », dans G. Schultz, dir., Trasparente Welt, Stuttgart, Hans Huber, p. 291-313. 
(1967), «Über den Sprachgebrauch Meister Eckharts: Gott muss... Ein Beispiel für die Gedankgänge der spekulativen Mystik », dans G. MülleR und W. Zeller, dir., Glaube, Geist, Geschichte. Festschrift für Ernst Benz, Leiden, E.J. Brill, p. 266-277.

(1977), «Das Nichts bei Meister Eckhart und im Zen-Buddhismus unter besonderer Berücksichtigung des Grenzbereich von Theologie und Philosophie ", dans Transzendenz und Immanenz. Philosophie und Theologie in der veränderten Welt, Internationale Zusammenarbeit in Grenzbereich von Philosophie und Theologie, Stuttgart, Kohlhammer, p. 257-266.

(1989), "Eckhart und Zen am Problem Freiheit und Sprache", Beihefte der Zeitschrift für Religions-und Geistsgeschichte, Joachim Wach Vorlesungen der Philipps Univ. Marburg, Köln, J. Brill, p. 21-92.

\section{Résumé}

Au début des années 1960, Ueda Shizuteru alla étudier à Marburg, sous la direction d'Ernst Benz. Le travail de cette époque se concentra sur la spéculation de Maître Eckhart et sur la comparaison avec le bouddhisme. En particulier, l'étude de Ueda fit émerger deux différentes approches de la question du néant. La mystique du néant et la śūnyatā bouddhiste furent analysées dans leurs aspects constitutifs. Cet approfondissement se révèle précieux, non seulement comme étude interculturelle, mais surtout comme contribution à l'interprétation du contexte nihiliste que l'Europe et le Japon se retrouvent à partager et auquel ces derniers ont affaire. Les observations de Ueda et, en général, des auteurs de l'École de Kyōto, offrent une perspective totalement autre dans la façon de traiter les nœuds ontologiques et théologiques de la pensée occidentale.

\section{Abstract}

In the early '60 Ueda Shizuteru went to study in Marburg, under the tutorship of Ernst Benz. In those years his work focused on Meister Eckhart's speculation and the comparison with Buddhism. Namely Ueda's study reveals two different approaches to the question of nothingness. Mystics of nothingness and Buddhist śūnyatā are analyzed in their constitutive aspects. This in-depth examination will prove valuable, not only as intercultural study, but also as a contribution to the interpretation of the nibilistic context that Europe and Japan are sharing and must face. Ueda's observations, as well as those of other authors of the Kyōto School, offer a totally different perspective in approaching the ontological and theological cruces of the western thought. 